\title{
Características elétricas e eficiência energética de um sistema de descarga de barreira dielétrica
}

\section{Electrical characteristics and energy efficiency of a dielectric barrier discharge system}

Clodomiro Alves Júnior ${ }^{1}$, Thomas Tadeu de Oliveira Pereira², Reuber Regis de Melo ${ }^{3}$, Hugo Fernandes Medeiros Silva ${ }^{4}$ Júlio César Pereira Barbosa ${ }^{1}$

\section{ABSTRACT}

The electrical, optical and energetic efficiency characteristics of an asymmetrical surface dielectric barrier discharge (DBD) in atmospheric air have been investigated experimentally. Voltages in the range of $18-29 \mathrm{kV}$ and $18-22 \mathrm{kHz}$ frequencies were applied to the DBD system, producing filamentous and diffuse discharges on the surface of a glass dielectric. These discharges reached peak currents up to $4 \mathrm{~mA}$. In terms of energetic efficiency, the system obtained minimum power $5 \mathrm{~W}$ required to produce plasma diffuse regime. The best conditions in the system were found for the distance between electrodes $0.8 \mathrm{~mm}$.

Keywords: Dielectric barrier discharge; Plasma applications; Plasma devices; Atmospheric plasma.

\section{RESUMO}

As características elétricas, ópticas e de eficiência energética de uma descarga de barreira dielétrica (DBD) com eletrodos assimétricos em ar atmosférico foram investigadas experimentalmente. As tensões na faixa de $18-29 \mathrm{kV}$ e frequência de 18-22 kHz foram aplicadas ao sistema DBD, produzindo descargas filamentosas e difusas na superfície de um dielétrico de vidro. Essas descargas atingiram correntes de pico até $4 \mathrm{~mA}$. Em termos de eficiência energética, o sistema obteve potência mínima de $5 \mathrm{~W}$ necessária para produzir um regime difuso de plasma. As melhores condições do sistema foram encontradas para a distância entre eletrodos de 0,8 mm

Palavras-chave: Descarga de barreira dielétrica; Aplicações de plasma; Dispositivo de plasma; Plasma em pressão atmosférica.

\footnotetext{
'Universidade Federal Rural do Semi-árido - Departamento de Ciências Exatas e Naturais - Mossoró (RN) - Brasil

2Universidade Federal Rural do Semi-árido - Programa de Pós-graduação em Ciência da Computação - Mossoró (RN) - Brasil

${ }^{3}$ Universidade Federal do Rio Grande do Norte - Programa de Pós-graduação em Engenharia Elétrica e de Computação - Natal (RN) - Brasil

${ }^{4}$ Universidade Federal do Rio Grande do Norte - Programa de Pós-graduação em Engenharia Mecânica - Natal (RN) - Brasil

Autor correspondente: Clodomiro Alves Júnior - Universidade Federal Rural do Semi-árido - Departamento de Ciências Exatas e Naturais - Av. Francisco

Mota, 572, Bairro Costa e Silva - CEP: 59.625-900 - Mossoró (RN) - Brasil

Email: alvesjr@pq.cnpq.br

Recebido: 12/09/2017 Aceito: 22/11/2017
} 


\section{INTRODUÇÃO}

Estudos na área de plasma em pressão atmosférica estão em crescente destaque devido sua versatilidade de aplicações e flexibilidade construtiva, ser ambientalmente limpo e possuir custo relativamente baixo de implantação. A técnica de plasma atmosférico conhecida como Descarga por Barreira Dielétrica (DBD) vem sendo usada em vários processos, como ativação e limpeza de superfícies, tratamento de materiais, esterilização de material hospitalar e tratamento médico ${ }^{(1)}$. No ramo alimentício pode-se destacar o uso do plasma para descontaminação de alimentos $^{(2)}$. Na agricultura estudos mostram a aplicação de plasma para inativação de fungos e bactérias, assim como o pré-tratamento de sementes para estimular a germinação $0^{(3-8)}$.

O DBD ocorre quando uma alta tensão pulsada é aplicada entre dois eletrodos metálicos, tendo entre eles uma barreira dielétrica. Quando a tensão rompe essa barreira dielétrica, várias microdescargas se distribuem na superfície do dielétrico dando origem ao plasma DBD em regime filamentar. Quando essas microdescargas são mais numerosas, homogêneas e distribuídas dão origem ao plasma DBD em regime difuso ${ }^{(9)}$. Algumas propriedades básicas do DBD e aplicações em tratamentos de superfícies podem ser visto no artigo "The barrier discharge: basic properties and applications to surface treatment"(10).

O plasma DBD pode ser caracterizado de acordo com a configuração e distribuição dos eletrodos. Eles podem ser configurados de maneira planar ou co-planar (disposição assimétrica). No primeiro caso a descarga acontece no volume entre os eletrodos, enquanto no segundo caso a descarga acontece na superfície de um deles, ou de ambos. E podem ser distribuídos cilindricamente para gerar jatos de plasma ou outra forma geométrica conveniente para a aplicação. Os materiais tipicamente usados para a barreira dielétrica são o vidro, quartzo e cerâmica. Também podem ser usados como material dielétrico: folhas de plástico, placas de PTFE-Politetrafluoretileno e outros materiais isolantes ${ }^{(10)}$.

No caso específico do plasma DBD na forma de descarga em superfície, existe uma larga faixa de tensão e de frequência possíveis para o surgimento da descarga ${ }^{(11)}$. Essa flexibilidade de variáveis do processo, aliada às características de impedância do sistema, tem causado grande dificuldade e confusão na análise da influência dos parâmetros do processo sobre a aplicação particular ${ }^{(12)}$. Um estudo de um atuador aerodinâmico a plasma, relatando a influência dos materiais dielétricos, distância entre eletrodos e a influência da frequência e tensão aplicada na potência dissipada pelo atuador, foi realizado na literatura ${ }^{(13)}$. Outros trabalhos existem na literatura envolvendo estudos sobre sistemas DBD assimétricos, onde a caracterização elétrica é uma forte fonte de conhecimento para ter o controle desses tipos de sistemas ${ }^{(14-17)}$. Entretanto, outro fator importante e que até o momento não foi considerado na literatura, são as densidades das espécies ativas produzidas pelo sistema, as quais são responsáveis pela interação do plasma.

Este trabalho apresenta um estudo que tem como objetivo entender, além dos parâmetros elétricos como energia por ciclo e potência dissipada, quanto de energia foi utilizada especificamente para produção das espécies ativas do plasma. A contribuição deste trabalho visa também explicar os resultados obtidos por Pereira, T.T.O. et al. ${ }^{(18)}$, onde houve um resultado positivo na molhabilidade e germinação quando sementes de "Sabiá" (Mimosa caesalpiniifolia Benth.) foram submetidas à presença do plasma por diferentes períodos de tempo.

\section{MATERIAIS E MÉTODOS}

O sistema DBD apresentado neste trabalho produz uma descarga na superfície de uma placa dielétrica plana, com dois eletrodos entre a placa e configurados assimetricamente, como é ilustrado na Fig. 1. Um dos eletrodos é conectado a uma fonte de alta tensão que fornece uma forma de onda senoidal da tensão. O eletrodo oposto é ligado ao terra, com uma resistência ou capacitância em série. $\mathrm{O}$ dielétrico é feito de vidro com uma espessura de $1.2 \mathrm{~mm}$. Os eletrodos consistem de finas tiras de cobre de comprimento $33 \mathrm{~mm}$, largura $11 \mathrm{~mm}$ e uma distância de $0.4 \mathrm{~mm}$ a $1.6 \mathrm{~mm}$ entre eletrodos. A tensão senoidal com amplitude máxima próxima de $15 \mathrm{kV}$, ou seja, $30 \mathrm{kV}$ pico a pico, é aplicada entre os eletrodos com uma frequência de $18-22 \mathrm{kHz}$.

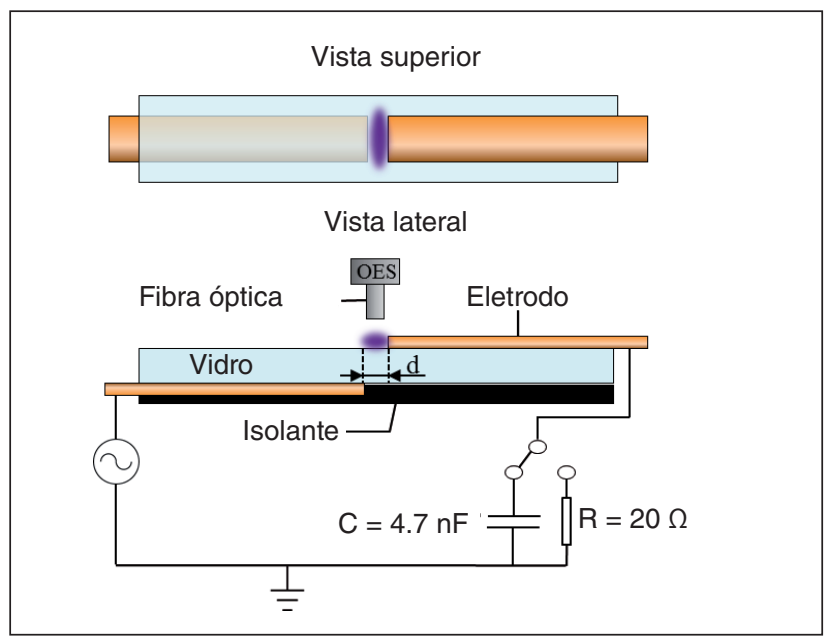

Figura 1: Diagrama do aparato experimental usado neste trabalho mostrando a configuração dos eletrodos coplanares e arranjo experimental para obtenção dos espectros ópticos e obtenção das curvas de lissajous.

As medidas elétricas do sistema DBD foram feitas usando um osciloscópio de dois canais (Keysight DSOX2002A). Os dados capturados pelo osciloscópio foram armazenados em um dispositivo de memória removível, e posteriormente visualizados graficamente no PC pelo software Origin ${ }^{\circledR}$ 8. Para medir a diferença de potencial aplicada sobre o sistema foi usada uma ponta de prova de alta tensão 1000:1 (Keysight N2771B). A 
corrente $i$ em função do tempo na descarga foi calculada pela Eq.1 de acordo com a Lei de Ohm, onde foi medida a queda de tensão $V_{R}$ sobre um resistor $R$ de $20 \Omega$ em série com o sistema. Para a medida dessa queda de tensão usou-se uma ponta de prova 10:1 (Keysight N2871A).

$$
i(t)=\frac{V_{R}(t)}{R}
$$

A carga transferida por cada ciclo de descarga foi medida experimentalmente usando um capacitor $C$ de $4.7 \mathrm{nF}$ em série ao sistema. A tensão $V_{C}$ sobre o capacitor é medida pela ponta de prova 10:1, e o valor da quantidade de carga $Q$ no capacitor é calculado pela Eq. 2.

$$
Q(t)=C V_{C}(t)
$$

Com essa medida de carga foi possível calcular a potência consumida pela descarga usando o método da figura de Lissajous, que consiste em plotar a carga transferida em função da tensão periódica aplicada. Baseado nos estudos de Rosenthal e Donald ${ }^{(19)}$, a energia elétrica $E$ consumida no processo de descarga por barreira dielétrica é dada pelo cálculo da área interna da figura de Lissajous, descrita por:

$$
E=\int_{0}^{t} V(t) d Q
$$

Onde $V$ é a tensão aplicada nos eletrodos e $d Q$ a carga infinitesimal do capacitor C. Dessa forma, a potência $P$ é obtida multiplicando a energia elétrica pela frequência do pulso de tensão aplicada, como demonstrado na Eq. 4.

$$
P=f E
$$

A área absoluta da figura de Lissajous foi calculada de forma computacional usando a ferramenta matemática de integração do programa Origin ${ }^{\circledR} 8$.

\section{Espectroscopia de Emissão Óptica}

Para a análise da intensidade luminosa do plasma gerado foram realizadas medições de Espectroscopia de Emissão Óptica (OES, do inglês Optical Emission Spectroscopy). O equipamento utilizado para essas medições foi um espectrômetro modelo USB4000 UV-VIS (Ocean Optics) juntamente com o software SpectraSuite. A aquisição do espectro foi obtida projetando a fibra óptica do equipamento aproximadamente $1 \mathrm{~mm}$ do plasma gerado pelo sistema DBD, como ilustrado na vista lateral da Fig. 1.

A intensidade luminosa $I$ proveniente do plasma atmosférico foi calculada pela Eq. 5, que basicamente significa a área sob a curva do espectro luminoso ${ }^{(1,20)}$. As medidas de OES são interessantes para identificar espécies atômicas, moleculares e íons formados no plasma. As principais espécies ativas típicas no DBD são as da molécula de, e se concentram mais na faixa de $300 \mathrm{~nm}$ a $420 \mathrm{~nm}^{(21)}$.

$$
I=\int_{300}^{420} I(\lambda) d \lambda
$$

Também foi calculada a eficiência do sistema DBD a partir da razão $I / E$. Essa medida permitiu visualizar qual a frequência e distância entre eletrodos tornavam o sistema DBD mais eficiente.

Por fim, a área sob a curva representada pela a integral da Eq. 5 também foi calculada de forma computacional com o auxílio do programa Origin ${ }^{\circledR} 8$.

\section{RESULTADOS E DISCUSSÕES}

A produção de plasma precisa de condições e parâmetros ideais para que ele seja formado. No estudo deste trabalho, o sistema DBD foi submetido a diferentes tensões, frequências e distâncias entre eletrodos. A seguir são apresentados os resultados de cada um dos parâmetros estudados.

O primeiro parâmetro estudado foi a influência da frequência da tensão aplicada sobre os eletrodos do sistema DBD, que serviu como base para as próximas análises. Nesse estudo, inicialmente manteve-se fixa uma tensão máxima fornecida pela fonte de tensão $\left(V=29 k V_{p-p}\right)$, e variou-se a frequência dessa tensão de 18-22 kHz. Essa faixa de frequência foi encontrada por meio de observações em testes experimentais.

A análise foi iniciada com a aplicação da tensão fixa para o eletrodo com distância $d=1.6 \mathrm{~mm}$ e frequência $f=18 \mathrm{kHz}$, onde foi observado o início da formação de plasma no sistema DBD. Posteriormente foi aplicada a mesma tensão fixa com frequências de $20 \mathrm{kHz}$ e $22 \mathrm{kHz}$. Dessa mesma forma foi analisada a geração de plasma para as distâncias de $0.4 \mathrm{~mm}, 0.8 \mathrm{~mm}$ e $1.2 \mathrm{~mm}$. Na Fig. 2 são mostradas as fotos dos diferentes plasmas gerados, sendo notória a influência da frequência e distância no comportamento do plasma. A partir desse estudo inicial foram feitas as caracterizações elétrica e óptica do sistema DBD, assim como a eficiência energética provocada pelas mudanças de parâmetros.

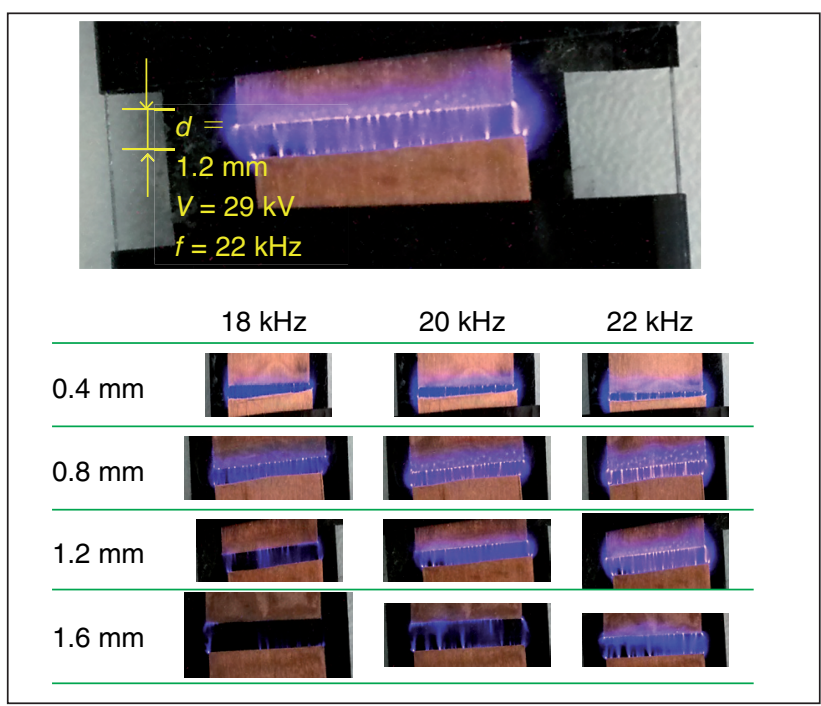

Figura 2: Comparação dos diferentes eletrodos submetidos a uma tensão fixa de $29 \mathrm{kV}_{p-p}$ com frequências de $18 \mathrm{kHz}, 20 \mathrm{kHz}$ e $22 \mathrm{kHz}$. 


\section{Caracterização Elétrica}

$\mathrm{Na}$ caracterização elétrica, a corrente na descarga foi calculada para as quatro distâncias estudadas, mantendo-se constante a tensão e frequência em $V=29 k V_{p-p}$ e $f=22 \mathrm{kHz}$. Na medida em que a distância entre os eletrodos aumenta, a amplitude da corrente na descarga diminui em decorrência do aumento da resistência. Para a distância de $0,4 \mathrm{~mm}$ o gráfico da Fig. 3 mostra a corrente com diversos picos bem acentuados, isto caracteriza a formação de arcos elétricos entre os eletrodos, devido à proximidade e a alta tensão aplicada.

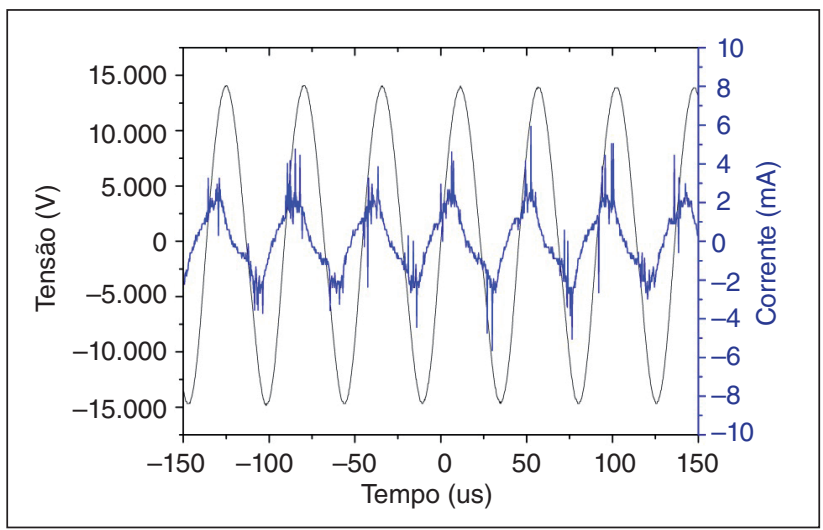

Figura 3: Tensão e corrente versus tempo $\left(V=29 \mathrm{kV}_{\mathrm{p}-\mathrm{p}}, f=22 \mathrm{kHz}\right.$, $d=0.4 \mathrm{~mm}$ )

Também na Fig. 3 pode ser observada uma pequena defasagem da corrente quando comparada com o sinal de tensão, causada pelo efeito capacitivo entre os eletrodos e igualmente observado nos estudos de Pons, J. ${ }^{(11)}$, Takashima, K. ${ }^{(22)}$ e Forte, M. ${ }^{(23)}$. Já na distância de 1,6 $\mathrm{mm}$ a amplitude da corrente é a menor como também a presença de arcos elétricos.

Outro parâmetro analisado foi a energia consumida por ciclo de produção de plasma para as quatro distâncias estudadas. Essa energia foi calculada pela área da figura de Lissajous e representada pela Eq. 3. A frequência de $22 \mathrm{kHz}$ apresentou maior energia em relação as demais, então foram analisados os gráficos de Lissajous para as diferentes distâncias nesta frequência, como pode ser observado na Fig. 4. Notou-se que com o aumento da distância

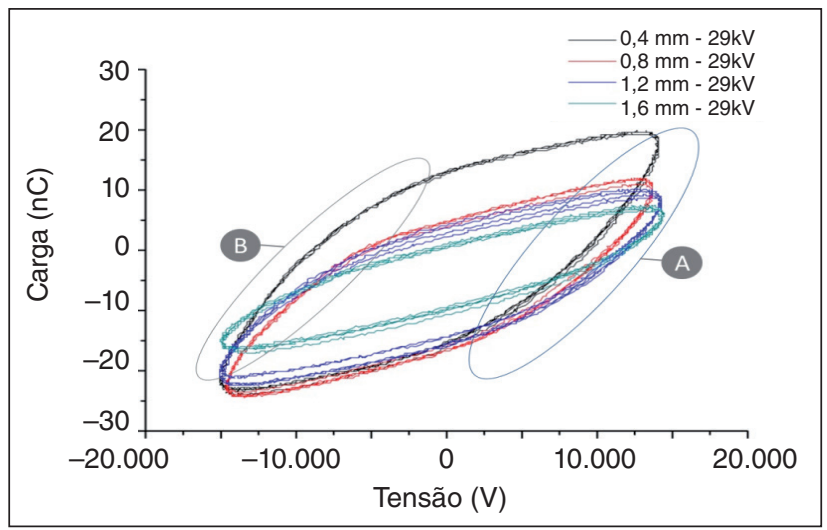

Figura 4: Figuras de Lissajous para $V=29 \mathrm{kV}_{\mathrm{p}-\mathrm{p}}, f=22 \mathrm{kHz}$ e distância $d$ de $0.4 \mathrm{~mm}$ a $1.6 \mathrm{~mm}$. entre os eletrodos ocorre a diminuição da energia consumida. O mesmo comportamento foi constatado para as frequências de $18 \mathrm{kHz}$ e $20 \mathrm{kHz}$, só que em proporções menores.

Analisando as figuras de Lissajous da Fig. 4, as regiões A e $B$ representam as descargas que produzem o plasma no sistema $\mathrm{DBD}^{(11,24)}$. É possível perceber que com o aumento da distância entre os eletrodos essas regiões sofrem uma considerável diminuição, provocando um afinamento da figura de Lissajous. Isso significa que menos descargas são geradas com o distanciamento dos eletrodos, sendo perceptível também nas imagens da Fig. 2.

Para todos os parâmetros mostrados na Fig. 2 foram medidas as energias através da figura de Lissajous, e posteriormente calculadas as potências de consumo para cada um usando a Eq. 4. Essas medidas são mostradas resumidamente na Fig. 5. O que se observou foi que assim como nos estudos apresentados por Pons, J. ${ }^{(11)}$, Takashima, K. ${ }^{(22)}$ e Forte, M. ${ }^{(23)}$, o aumento da frequência provocou um aumento com característica linear da potência consumida para todas as distâncias estudadas. Verificou-se também que quanto menor a distância entre eletrodos, maior é a potência consumida.

No trabalho de Roth e Jin ${ }^{(13)}$, também se verifica um comportamento de aumento de potência quando um atuador aerodinâmico a plasma, denominado One Atmosphere Uniform Glow Discharge Plasma (OAUGDP®), é submetido a aumento de frequência da tensão aplicada. Nesse atuador dois dos dielétricos testados pelo autor são de PTFE-Politetrafluoretileno e Quartzo. É preciso salientar que OAUGDP apresentado por Roth e Jin ${ }^{(13)}$ possui eletrodos de tamanhos diferentes aos estudados neste trabalho, mas a disposição dos mesmos está em conformidade muito próxima ao mostrado na Fig. 1.

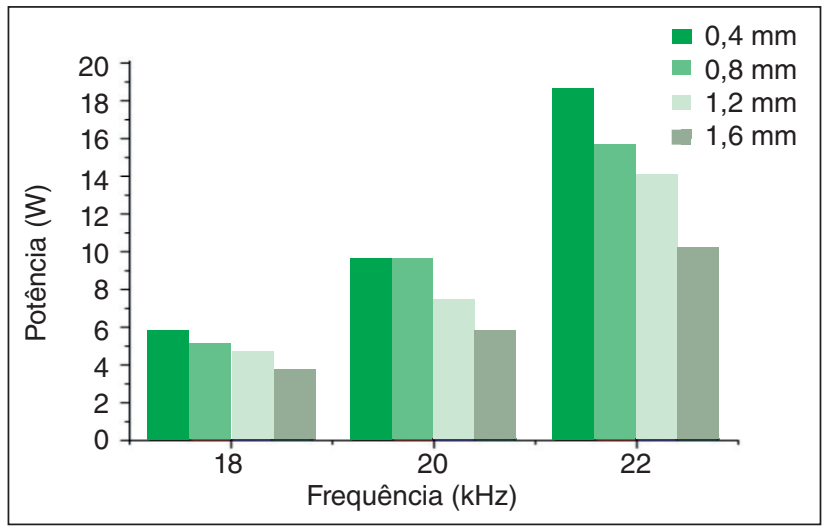

Figura 5: Potência consumida do sistema DBD em função da frequência e distância entre eletrodos $\left(V=29 \mathrm{kV}_{\mathrm{p} \text { p }}\right)$.

Na Fig. 6 foi analisada a potência consumida do sistema para três valores de tensão aplicada com frequência fixa de $22 \mathrm{kHz}$. Com essa análise verificou-se que a potência consumida aumenta rapidamente com o aumento da tensão, evidenciando uma maior produção de microdescargas sobre o sistema DBD. Nessa mesma análise, quanto menor a distância entre eletrodos também é maior a potência consumida. 
Na comparação com a Fig. 5 é possível ajustar uma mesma potência trabalhando com uma tensão baixa e uma frequência alta. Por exemplo, se um sistema DBD com eletrodos separados a uma distância de $0.8 \mathrm{~mm}$ precisasse trabalhar com uma potência de $3 \mathrm{~W}$, com base na figura Fig. 5 seria preciso uma tensão de e uma frequência abaixo de $18 \mathrm{kHz}$. Por outro lado, analisando a Fig. 6 para esse mesmo exemplo, seria preciso uma tensão pouco menor que e uma frequência de $22 \mathrm{kHz}$. Por questão de segurança na isolação do sistema é preferível trabalhar com tensões menores e, portanto, ter uma fonte de tensão com maior frequência.

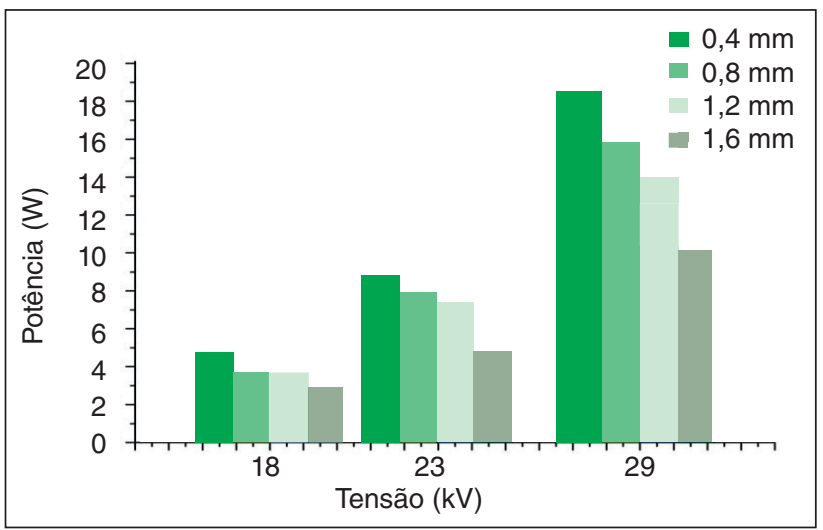

Figura 6: Potência consumida do sistema DBD em função da tensão aplicada e distância entre eletrodos ( $f=22 \mathrm{kHz})$.

\section{Caracterização Óptica}

Além da caracterização elétrica, foi realizada a caracterização óptica do sistema DBD com o intuito de analisar as espécies ativas do plasma gerado. Primeiramente observou-se o efeito da frequência na intensidade espectral. Na Fig. 7 é mostrado o espectro luminoso obtido por OES para o sistema DBD com a distância entre eletrodos de $0.8 \mathrm{~mm}$. Nota-se que o espectro possui picos mais intensos na medida em que a frequência aumenta. Também foram verificados os espectros para as outras distâncias, mas constatou-se que os picos eram menores que os apresentados na Fig. 7.

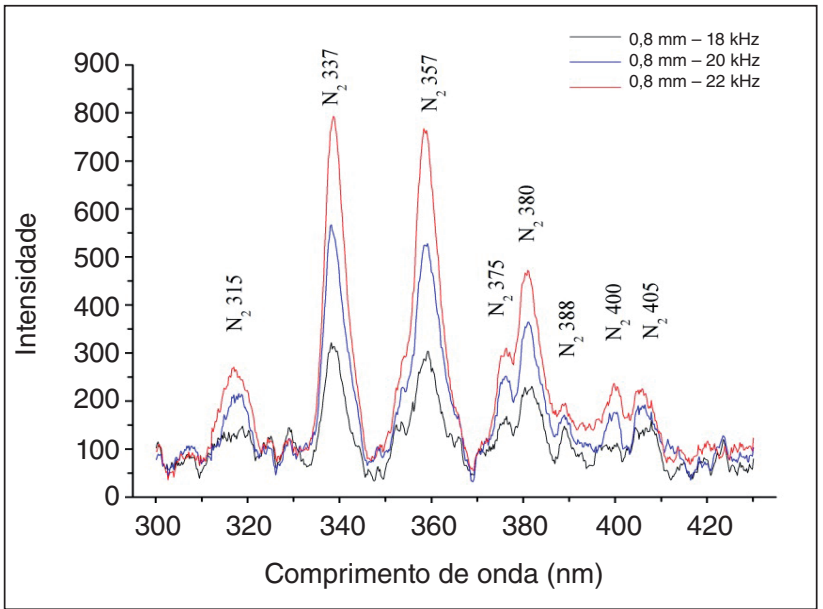

Figura 7: OES para o sistema DBD com e submetido a diferentes frequências $\left(29 \mathrm{kV}_{\mathrm{p}-\mathrm{p}}\right)$.
Relacionando a intensidade luminosa com potência consumida, verifica-se que a condição com maior energia consumida é também a que tem maior intensidade, o que é um indício de que a energia foi utilizada principalmente para ionização do ar atmosférico.

Na análise de OES, os picos em destaques foram os do $N_{2}(337 \mathrm{~nm})$ e $N_{2}(357 \mathrm{~nm})$, seguidos de seis outros picos, todos na região UV. Estes resultados estão coerentes com aqueles obtidos na literatura "Spectroscopic study of dielectric barrier discharges in cellular polypropylene ferroelectrets"(25).

Por fim, é importante ter o controle desses parâmetros para um bom desempenho de um processo que venha utilizar esse sistema DBD.

Na Fig. 8 são observados os espetros do sistema para as quatro distâncias estudadas em uma frequência de $22 \mathrm{kHz}$, e é verificado que a distância $0.8 \mathrm{~mm}$ possui maior intensidade. É interessante observar que ao verificar a Fig. 5 , a distância $0.4 \mathrm{~mm}$ na frequência de $22 \mathrm{kHz}$ possui maior potência consumida, porém, na Fig. 8 possui a menor intensidade luminosa. Isso revela que boa parte do gasto energético não está sendo gasto para ionização do ar, mas provavelmente na forma de calor pela resistência do dielétrico. As distâncias $1.2 \mathrm{~mm}$ e $1.6 \mathrm{~mm}$ possuem intensidades bem próximas, mas o que se percebe é que o aumento da distância acima de $1 \mathrm{~mm}$ leva a uma perda de intensidade luminosa.

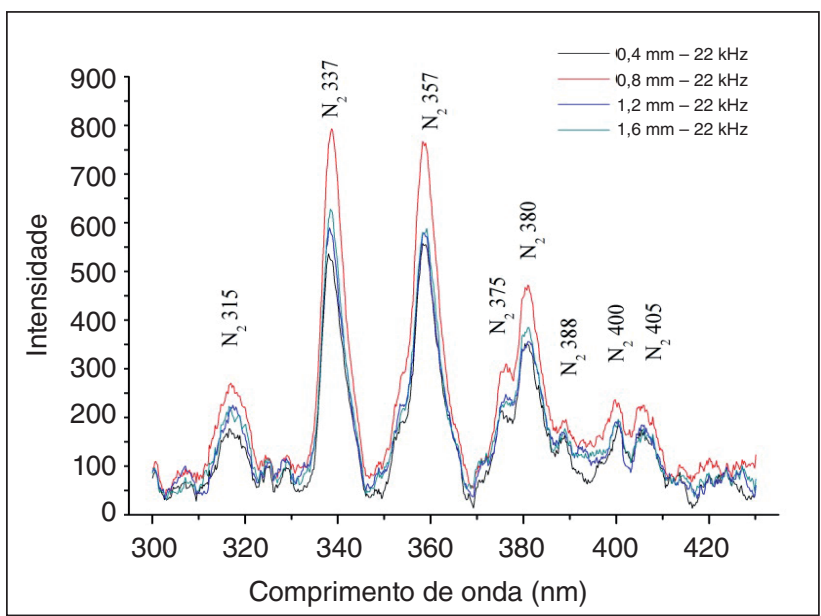

Figura 8: OES para o sistema DBD $\operatorname{com} f=22 \mathrm{kHz}$ e diferentes distâncias entre eletrodos $\left(V=29 \mathrm{kV}_{\mathrm{p}-\mathrm{p}}\right)$.

\section{Eficiência do Sistema DBD}

Tendo os valores de energia e intensidade dos plasmas gerados, foi calculada a eficiência do sistema DBD fazendo a razão entre a intensidade e energia consumida. Os valores de eficiência estão contidos na última coluna da Tabela 1 . A configuração que se apresentou mais eficiente foi a de distância $0.8 \mathrm{~mm}$ entre os eletrodos para frequência de $18 \mathrm{kHz}$, já para a frequência de $22 \mathrm{kHz}$ apesar de apresentar os maiores valores de energia e intensidade, não se demonstrou tão eficiente.

Para as três frequências estudadas, a distância de $0.8 \mathrm{~mm}$ apresentou a maior intensidade luminosa gerada. Em algumas 
Tabela 1: Parâmetros e medidas analisadas

\begin{tabular}{|c|c|c|c|c|c|c|}
\hline $\mathrm{d}(\mathrm{mm})$ & $\mathbf{f}(\mathrm{kHz})$ & $V(k V p-p)$ & $E(\mu J)$ & $P(W)$ & I & Eficiência (I/E) \\
\hline 0.4 & 18 & 29 & 311 & 5,6 & 12908 & 41 \\
\hline 0.8 & 18 & 29 & 281 & 5,1 & 14930 & 53 \\
\hline 1.2 & 18 & 29 & 241 & 4,3 & 12292 & 51 \\
\hline 1.6 & 18 & 29 & 214 & 3,8 & 10194 & 48 \\
\hline 0.4 & 20 & 29 & 486 & 9,7 & 16925 & 35 \\
\hline 0.8 & 20 & 29 & 482 & 9,6 & 21175 & 44 \\
\hline 1.2 & 20 & 29 & 365 & 7,3 & 17090 & 47 \\
\hline 1.6 & 20 & 29 & 290 & 5,8 & 15350 & 530 \\
\hline 0.4 & 22 & 29 & 846 & 18,6 & 18356 & 22 \\
\hline 0.8 & 22 & 29 & 715 & 15,7 & 26872 & 38 \\
\hline 1.2 & 22 & 29 & 640 & 14,1 & 20671 & 32 \\
\hline 1.6 & 22 & 29 & 460 & 10,1 & 21158 & 46 \\
\hline
\end{tabular}

situações a distância $1.6 \mathrm{~mm}$ obteve melhor eficiência, como no caso das frequências de $20 \mathrm{kHz}$ e $22 \mathrm{kHz}$. Além de uma boa eficiência, outro fator importante no uso de sistemas DBD é a geração de plasma no regime difuso. Esse regime é o mais indicado para a maioria das aplicações industriais devido a sua capacidade de tratamento superficial mais uniforme ${ }^{(26)}$. Dentre os parâmetros usados neste trabalho, a potência mínima necessária ao surgimento do plasma DBD em regime difuso é 5 $\mathrm{W}$, obtida na frequência $18 \mathrm{kHz}$ e com $0.8 \mathrm{~mm}$ de distância entre eletrodos.

\section{CONCLUSÃO}

Este estudo tem ajudado a compreender o comportamento elétrico de um sistema DBD em pressão atmosférica e na caracterização óptica das espécies ativas do plasma gerado. $\mathrm{O}$ sistema DBD produziu picos de corrente na faixa de $4 \mathrm{~mA}$ e potência média de $9.1 \mathrm{~W}$. De modo geral a energia por ciclo de descarga é maior para menores distâncias entre eletrodos. As principais espécies ativas na descarga por barreira dielétrica foram da molécula de , com maiores intensidades na distância $0.8 \mathrm{~mm}$. A melhor condição encontrada no presente estudo foi na distância $0.8 \mathrm{~mm}$ com frequência de $18 \mathrm{kHz}$, devido ao fato de obter melhor eficiência do sistema DBD e a produção de plasma em regime difuso.

Apesar de um sistema pequeno e simples, o sistema DBD apresentado por este trabalho tem grande potencial para trabalhos futuros, onde poderá ser expandido para criar sistemas DBDs mais complexos. Por exemplo, é possível montar ao longo de uma superfície plana e retangular vários eletrodos assimétricos paralelamente para percorrer sobre essa superfície materiais a serem tratados pelo plasma gerado. Essa ideia vem sendo explorada pelo Labplasma da UFERSA em busca de um tratamento para sementes do semiárido brasileiro.

\section{AGRADECIMENTOS}

Os autores agradecem o apoio estrutural da UFERSA e o apoio financeiro do CNPQ - Conselho Nacional de Desenvolvimento Científico e Tecnológico (Proc. 403230/2013-6, Proc. CNPq465423/2014-0 e Proc. CNPq-465423/2014-0) e CAPES (bolsas).

\section{REFERÊNCIAS}

1. KUCHENBECKER, M. et al., Characterization of DBD plasma source for biomedical applications. Journal of Physics D: Applied Physics 42.4 (2009): 045212

2. MISRA, N. N. et al., In-package atmospheric pressure cold plasma treatment of strawberries. Journal of Food Engineering 125 (2014): 131-138.

3. FILATOVA, I, et al., The effect of plasma treatment of seeds of some grain and legumes on their sowing quality and productivity. Romanian Journal of Physics 56 (2011): 139-143.

4. MITRA, A. et al., Inactivation of surface-borne microorganisms and increased germination of seed specimen by cold atmospheric plasma. Food and Bioprocess Technology 7.3 (2014): 645-653.

5. DUBINOV, A. E.; LAZARENKO,E.R.; SELEMIR, V.D., Effect of glow discharge air plasma on grain crops seed. IEEE transactions on plasma science 28.1 (2000): 180-183.

6. ŠERÁ, B. et al., How various plasma sources may affect seed germination and growth. Optimization of Electrical and Electronic Equipment (OPTIM), 2012 13th International Conference on IEEE, 2012.

7. ŠERÁ, B. ET al., Influence of plasma treatment on wheat and oat germination and early growth. Plasma Science, IEEE Transactions on 38.10 (2010): 2963-2968.

8. ZHOU, Z. et al., Introduction of a new atmospheric pressure plasma device and application on tomato seeds. Agricultural Sciences 2.01 (2011): 23

9. PIETSCH, G. J., Peculiarities of dielectric barrier discharges Contributions to Plasma Physics 41.6 (2001): 620-628.

10. WAGNER, H-E. ET al., The barrier discharge: basic properties and applications to surface treatment. Vacuum 71.3 (2003): 417 436. 
11. PONS, J.; ERIC, M.; GÉRARD, T., Asymmetric surface dielectric barrier discharge in air at atmospheric pressure: electrical properties and induced airflow characteristics. Journal of Physics D: Applied Physics 38.19 (2005): 3635.

12. DOBRIN, D. ET AL., The effect of non-thermal plasma treatment on wheat germination and early growth. Innovative Food Science \& Emerging Technologies 29 (2015): 255-260.

13. ROTH, J.R.; XIN, D., Optimization of the aerodynamic plasma actuator as an electrohydrodynamic (EHD) electrical device. 44th AIAA Aerospace Sciences Meeting and Exhibit (2006).

14. KRIEGSEIS, J.; SVEN G.; CAMERON, T., Performance reduction of Dielectric Barrier Discharge plasma actuators at higher Mach numbers. New Results in Numerical and Experimental Fluid Mechanics VIII. Springer Berlin Heidelberg, 2013. 209-217.

15. ENLOE, C. L. ET AL., Mechanisms and responses of a single dielectric barrier plasma actuator: plasma morphology." AIAA journal 42.3 (2004): 589-594.

16. THOMAS, F. O. et al., Optimization of dielectric barrier discharge plasma actuators for active aerodynamic flow control. AIAA journal 47.9 (2009): 2169-2178.

17. HE, C.; THOMAS, C. C.; MEHUL P. P., Plasma flaps and slats: an application of weakly ionized plasma actuators. Journal of Aircraft 46.3 (2009): 864-873.

18. PEREIRA, T.T.O. et al., The effect of DBD plasma treatment of "Sabiá" (Mimosa caesalpiniifolia Benth.) seeds. XXXVI CBRAVIC / I WTMS (2015): 171.
19. ROSENTHAL, L. A.; DONALD, A. D., Electrical characterization of a corona discharge for surface treatment. Industry Applications, IEEE Transactions on 3 (1975): 328-335.

20. RAJASEKARAN, P. ET AL., Filamentary and homogeneous modes of dielectric barrier discharge (DBD) in air: investigation through plasma characterization and simulation of surface irradiation. Plasma Processes and Polymers 7.8 (2010): 665-675.

21. STAACK, D. et al., DC normal glow discharges in atmospheric pressure atomic and molecular gases. Plasma Sources Science and Technology 17.2 (2008): 025013

22. TAKASHIMA, K. et al., Characterization of a surface dielectric barrier discharge plasma sustained by repetitive nanosecond pulses. Plasma Sources Science and Technology 20.5 (2011): 055009.

23. FORTE, M. et al., Optimization of a dielectric barrier discharge actuator by stationary and non-stationary measurements of the induced flow velocity: application to airflow control. Experiments in Fluids 43.6 (2007): 917-928.

24. KRIEGSEIS, J. et al., Light Emission, Discharge Capacitance and Thrust Production of DBD Plasma Actuators. 49th AIAA Aerospace Sciences Meeting, AIAA. Vol. 155. 2011.

25. QIU, X. et al., Spectroscopic study of dielectric barrier discharges in cellular polypropylene ferroelectrets. Applied Physics Letters 91.13 (2007): 132905.

26. FANG, Z. et al., Comparison of surface modification of polypropylene film by filamentary DBD at atmospheric pressure and homogeneous DBD at medium pressure in air. Journal of Physics D: Applied Physics 42.8 (2009): 085204. 